\title{
Using interaction-based readouts to approach the ultimate limit of detection-noise robustness for quantum-enhanced metrology in collective spin systems
}

\author{
Simon A. Haine* \\ Department of Physics and Astronomy, University of Sussex, Brighton BN1 9QH, United Kingdom \\ and Department of Quantum Science, Australian National University, Canberra, Australia
}

(Received 31 May 2018; published 28 September 2018)

\begin{abstract}
We consider the role of detection noise in quantum-enhanced metrology in collective spin systems and derive a fundamental bound for the maximum obtainable sensitivity for a given level of added detection noise. We then present an interaction-based readout utilizing the commonly used one-axis twisting scheme that approaches this bound for states generated via several commonly considered methods of generating quantum enhancement, such as one-axis twisting, two-axis countertwisting, twist-and-turn squeezing, quantum nondemolition measurements, and adiabatically scanning through a quantum phase transition. We demonstrate that our method performs significantly better than other recently proposed interaction-based readouts. These results may help provide improved sensitivity for quantum-sensing devices in the presence of unavoidable detection noise.
\end{abstract}

DOI: 10.1103/PhysRevA.98.030303

There is a continued push for improved metrological potential in devices such as atomic clocks, atomic magnetometers, and inertial sensors based on atom interferometry [1]. The physics of these systems is well described by collective spin systems [2]. Over the past decade, there has been rapid progress in the demonstration of quantum-enhanced metrology in these systems, that is, parameter estimation with sensitivity surpassing the shot-noise limit (SNL) [3-19]. These schemes generally require a state preparation step, where interparticle entanglement is created to enhance the metrological potential [20-22], before the classical parameter of interest (which is usually proportional to a phase) is encoded onto the state. There exists a plethora of state preparation techniques for creating highly quantum enhanced states, such as quantum state transfer from light to atoms [23-32], quantum nondemolition measurement (QND) [4,18,33-36], spin changing collisions [10,11,37-39], one-axis twisting (OAT) [3,6,8,9,40-42], two-axis countertwisting (TACT) $[40,43]$, twist-and-turn squeezing (TNT) $[16,44]$, and adiabatically scanning through a quantum phase transition (QPT) [45-51]. However, the states generated via these schemes almost always require detection with very low noise (of the order of less than one particle) in order to see significant quantum enhancement $[2,52,53]$.

Recently, there has been considerable interest in the concept of interaction-based readouts (IBRs) [50,51,54-66], which are periods of unitary evolution applied to the system after the phase encoding step but before the measurement takes place. These readouts usually involve interparticle interactions, similar to the ones used for the state preparation. Davis et al. showed that by using OAT to prepare a state with high quantum Fisher information (QFI), applying a phase shift, and then employing an IBR that reverses the OAT dynamics, quantum-enhanced sensitivity could be achieved well

\footnotetext{
*simon.a.haine@gmail.com
}

beyond the Gaussian spin-squeezing regime. Furthermore, this quantum enhancement persisted even when the added detection noise was as large as the projection noise [54]. Similarly, Hosten et al. experimentally demonstrated that a period of nonlinear evolution after the state preparation and phase encoding could achieve sub-SNL sensitivity in the presences of significant detection noise [55]. Macri et al. demonstrated that by performing an IBR that perfectly reverses the state preparation and then projects into the initial state, the sensitivity saturates the quantum Cramér-Rao bound (QCRB) [57]. Nolan et al. [60] further generalized this result to show that there exist many IBRs that satisfy the conditions for saturating the QCRB and that the choice of IBR has implications for the level of sensitivity in the presence of detection noise (or "robustness"). In particular, it was found that the optimum IBR was not necessarily the one that perfectly reversed the state preparation. Furthermore, it was demonstrated that sensitivity approaching the Heisenberg limit $[67,68]$ could be achieved in the presence of detection noise approaching the number of particles. IBRs have also been explored by applying time reversal of the state-preparation dynamics in systems where the quantum-enhanced state is generated via SCC [58,59,69], TACT [62], TNT [64], and QPT [50,51].

In this work, we derive a limit for sensitivity in the presence of detection noise, which is significantly better than the levels achievable via previous schemes. We then present an IBR based on OAT that approaches this limit for states generated via OAT, TNT, TACT, QPT, and QND.

\section{ULTIMATE SENSITIVITY LIMIT IN THE PRESENCE OF DETECTION NOISE}

The sensitivity with which we can estimate the classical parameter $\phi$ is quantified via the Cramér-Rao bound: $\Delta \phi^{2}=$ $1 / F_{C}$, where $F_{C}$ is the classical Fisher information (CFI), defined by $F_{C}=\sum_{m} \dot{P}_{m}^{2} / P_{m}$, where $P_{m}$ is the probability of obtaining measurement result $m$ and $\dot{P}_{m} \equiv \partial_{\phi} P_{m}$. Assuming a 
collection of $N$ particles distributed amongst two modes, the natural description for our system is provided via the pseudospin $\mathrm{SU}(2)$ algebra: $\left[\hat{J}_{x}, \hat{J}_{y}\right]=i \hat{J}_{z}$ [70]. The eigenstates of these operators form a natural basis of easily accessible measurements, as they can be obtained via single-particle operations such as linear rotations and particle counting [2]. For simplicity, throughout this paper we assume that measurements are made by projecting into the $\hat{J}_{z}$ basis, i.e., $\{|m\rangle\langle m|\}$, where $\hat{J}_{z}|m\rangle=m|m\rangle$. The particular direction is of little consequence, however, as projections along other directions can be obtained via linear rotations. Following the convention introduced in Ref. [71] and subsequently used in Refs. [2,50,56,60,62,64,65,69], we model the behavior of an imperfect detector as sampling from the probability distribution

$$
\tilde{P}_{m}(\sigma)=\sum_{m^{\prime}} \Gamma_{m, m^{\prime}}(\sigma) P_{m^{\prime}}
$$

where

$$
\Gamma_{m, m^{\prime}}(\sigma)=e^{-\left(m-m^{\prime}\right)^{2} /\left(2 \sigma^{2}\right)} / \sum_{m} e^{-\left(m-m^{\prime}\right)^{2} /\left(2 \sigma^{2}\right)}
$$

introduces detection noise of magnitude $\sigma$. This is equivalent to the positive operator valued measurement (POVM) $\left\{\hat{M}_{m}\right\}=$ $\left\{\sum_{m^{\prime}} \Gamma_{m, m^{\prime}}\left|m^{\prime}\right\rangle\left\langle m^{\prime}\right|\right\}$. To demonstrate how the noise affects the CFI, we consider the case where $P_{m}$ contains only two nonzero elements, $P_{a}$ and $P_{b}$, with $P_{b}=1-P_{a}$, and $\dot{P}_{a}=$ $-\dot{P}_{b}=\sqrt{F_{0}\left(P_{a}-P_{a}^{2}\right)}$, such that $F_{C}=F_{0}$. By approximating $m$ as a continuous variable and extending the domain to $\pm \infty$ [72], we obtain

$$
\tilde{P}(m)=\left(P_{a} e^{-(a-m)^{2} / 2 \sigma^{2}}+P_{b} e^{-(b-m)^{2} / 2 \sigma^{2}}\right) / \sqrt{2 \pi} \sigma .
$$

Defining

$\tilde{P}_{a}=\int_{-\infty}^{\frac{1}{2}(a+b)} \tilde{P}(m) d m \quad$ and $\quad \tilde{P}_{a}=\int_{\frac{1}{2}(a+b)}^{\infty} \tilde{P}(m) d m$

(assuming $a<b)$ and maximizing with respect to $P_{a}\left(P_{a} \rightarrow\right.$ $P_{b} \rightarrow \frac{1}{2}$ ), we obtain

$$
F_{C}(\sigma)=\dot{\tilde{P}}_{a}^{2} / \tilde{P}_{a}+\dot{\tilde{P}}_{b}^{2} / \tilde{P}_{b} \approx F_{0}(\operatorname{Erf}[(a-b) / 2 \sqrt{2} \sigma])^{2} .
$$

Clearly, $F_{C}(\sigma)$ decays less rapidly when the separation between the nonzero components of $P_{m},|a-b|$ is large compared to $\sigma$. This intuition leads us to postulate that distribution with maximum robustness, $P_{\mathrm{opt}}$ is

$$
\begin{aligned}
& P_{N / 2}=P_{-N / 2}=\frac{1}{2}, \\
& \dot{P}_{N / 2}=-\dot{P}_{-N / 2}=\sqrt{F_{0}} / 2,
\end{aligned}
$$

with all other elements equal to zero. While an analytic proof of this remains elusive, we confirm this via a numeric optimization method [72]. In the absence of detection noise, the QCRB states that $F_{C} \leqslant F_{Q}$, where $F_{Q}$ is the QFI. We define the noisy $Q C R B$ (NQCRB) as $F_{C}(\sigma) \leqslant F_{n}(\sigma)$, where $F_{n}(\sigma)$ is the CFI calculated from the $\left\{\tilde{P}_{m}(\sigma), \dot{\tilde{P}}_{m}(\sigma)\right\}$ obtained from performing the discrete sum in Eq. (1) numerically with $\left\{P_{m}, \dot{P}_{m}\right\}=\left\{P_{\text {opt }}, \dot{P}_{\text {opt }}\right\}$ and setting $F_{0}=F_{Q}$. This is the maximum sensitivity that can be achieved by making spin measurements on a state with QFI equal to $F_{Q}$ in the

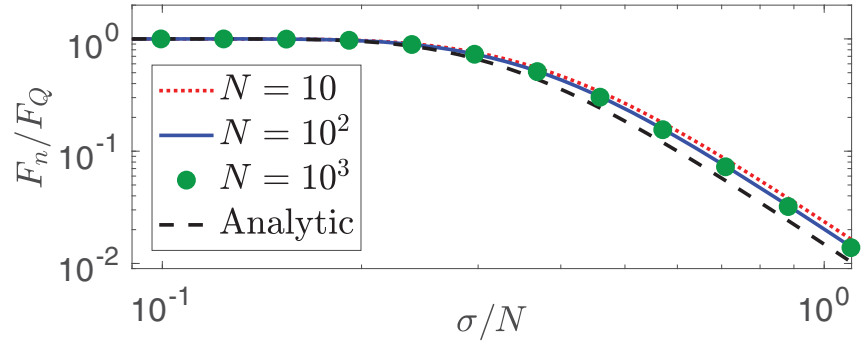

FIG. 1. The exact numeric value of $F_{n}$ vs $\sigma / N$ for $N=10,10^{2}$, and $10^{3}$, compared to the approximate expression Eq. (7). The shape of $F_{n}(\sigma / N)$ is almost identical for $N=10^{2}$ and $10^{3}$.

presence of detection noise $\sigma$. We can get an approximate analytic expression for $F_{n}(\sigma)$ by again approximating $m$ as a continuous variable, but limiting the range to $-N / 2<m<$ $N / 2$, such that

$$
F_{n}(\sigma) \approx F_{Q}\left(1-2 \frac{\operatorname{Erf}[\alpha / 2]}{\operatorname{Erf}[\alpha]}\right)^{2},
$$

with $\alpha=N / \sqrt{2} \sigma$. Figure 1 shows excellent agreement between this expression and the exact value of $F_{n}(\sigma)$, calculated numerically. Equation (7) provides a slight under-estimate of the CFI, as information is lost when condensing $P_{m}$ into a binary distribution via Eq. (4). For the remainder of this paper, we use the exact numeric value of $F_{n}(\sigma)$ rather than Eq. (7).

\section{INTERACTION-BASED READOUT TO SATURATE THE NQCRB}

The NQCRB sets the maximum achievable CFI in the presence of detection noise $\sigma$. What remains is to find an IBR that allows us to achieve this limit. Starting with an arbitrary initial pure state $\left|\psi_{1}\right\rangle$, we note that this state can always be written as $\left|\psi_{1}\right\rangle=U_{1}\left|\psi_{0}\right\rangle$, where $\left|\psi_{0}\right\rangle=\left|\frac{N}{2}\right\rangle$ is the maximal $\hat{J}_{z}$ eigenstate, which is completely separable in the particle basis. In most quantum enhanced metrology schemes, the unitary operator $U_{1}$ implements the state preparation step, which may be employed to increase the QFI of an initially separable state. Specific examples of this process including OAT, TACT, TNT, and QPT will be considered later. The phase shift $\phi$ is then encoded on to the state via $\left|\psi_{\phi}\right\rangle=$ $e^{i \hat{J}_{n} \phi}\left|\psi_{1}\right\rangle$, where $\hat{J}_{n}=\mathbf{J} \cdot \mathbf{n}$, and $\mathbf{n}$ is a unit vector chosen to maximise the QFI of $\left|\psi_{\phi}\right\rangle$. This vector can be obtained from the collective covariance matrix [20]. An IBR is some unitary $U_{2}$ such that measurements are made on the state $U_{2}\left|\psi_{\phi}\right\rangle$. Our goal is to find $U_{2}$ such that the probability distribution $P_{m}=\left|\left\langle m\left|U_{2}\right| \psi_{\phi}\right\rangle\right|^{2}$ saturates the NQCRB. It was shown in Ref. [57] that for $\phi \ll 1$, selecting $U_{2}=U_{1}^{\dagger}$ saturates the QCRB. At some value $\phi=\phi_{0}$,

$$
U_{1}^{\dagger} e^{i \hat{J}_{n} \phi_{0}} U_{1}\left|\psi_{0}\right\rangle=\frac{1}{\sqrt{2}}\left(\left|\psi_{0}\right\rangle+\left|\psi^{\prime}\right\rangle\right) \equiv\left|\psi_{b}\right\rangle,
$$

where

$$
\left|\psi^{\prime}\right\rangle=\left(\hat{1}-\left|\psi_{0}\right\rangle\left\langle\psi_{0}\right|\right)\left|\psi_{b}\right\rangle / \sqrt{1-\left|\left\langle\psi_{b} \mid \psi_{0}\right\rangle\right|^{2}} .
$$

We can artificially construct an IBR that is maximally robust to noise simply by constructing a unitary operator $U_{\mathrm{p}}$ that 

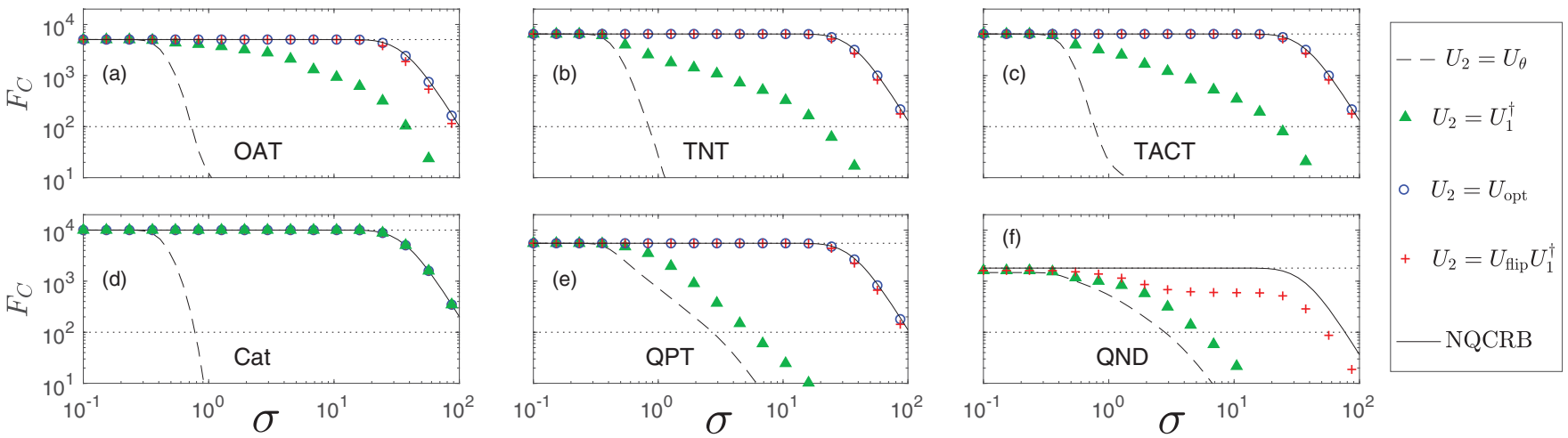

FIG. 2. $F_{C}(\sigma)$ for (a) OAT with $r=0.2$, (b) TNT, (c) TACT, (d) OAT with $r=\frac{\pi}{2}$ (which corresponds to a spin-cat state), (e) QPT, and (f) QND. $U_{\theta}=e^{i \frac{\pi}{2} \hat{\jmath}_{y}}$ for OAT, Cat, and TNT, and $U_{\theta}=1$ for TACT, QPT, and QND. The upper and lower dotted black lines indicate the QCRB $\left(F_{C}=F_{Q}\right)$ and SNL $\left(F_{C}=N\right)$, respectively. $N=100$ for all cases, and we have optimized over $\phi$. The optimum $\phi$ is close to $\phi_{0}$ for $U_{2}=U_{\text {opt }}$ and close to 0 for $U_{2}=U_{\text {flip }} U_{1}^{\dagger}$.

maps this state to one with distribution $P_{\mathrm{opt}}$ :

$$
U_{\mathrm{p}}=\left|\frac{N}{2}\right\rangle\left\langle\frac{N}{2}|+| \frac{-N}{2}\right\rangle\left\langle\psi^{\prime}\left|+\sum_{m=-N / 2+1}^{N / 2-1}\right| m\right\rangle\left\langle m^{\prime}\right|,
$$

where $\left\{\left|m^{\prime}\right\rangle\right\}$ completes the orthogonal basis containing $\left|\frac{N}{2}\right\rangle$ and $\left|\psi^{\prime}\right\rangle$. Thus, the optimum IBR is

$$
U_{2}=U_{\mathrm{p}} U_{1}^{\dagger} \equiv U_{\mathrm{opt}} .
$$

Figure 2 shows the CFI calculated from $P_{m}=\left|\left\langle m\left|U_{\text {opt }}\right| \psi_{\phi}\right\rangle\right|^{2}$ after convolving with detection noise, for quantum enhanced states generated from OAT, TACT, TNT, and QPT. Details of these states are provided in Table I [72]. In all cases, we find that this IBR saturates the NQCRB. To understand the mechanism for this, we consider the effect of detection noise on the probability distributions. Figure 3 shows $P_{m}(\phi)$ and $P_{m}(\phi+\delta \phi)$, with (right column) and without (left column) noise, for the case of OAT. When $U_{2}=U_{1}^{\dagger}$ [Figs. 3(a) and 3(e)], the change in probability is centered around $m=\frac{N}{2}$ and nearby elements. When detection noise is added, $P_{m}(\phi)$ and $P_{m}(\phi+\delta \phi)$ become less distinct as the adjacent elements are mixed. However, by applying $U_{2}=U_{\text {opt }}$ [Figs. 3(b) and 3(f)], all of the probability in elements $m \neq \frac{N}{2}$ is transferred to $m=-\frac{N}{2}$ such that $P_{m}=P_{\mathrm{opt}}$. We stress that the

TABLE I. Details of the quantum state $\left|\psi_{1}\right\rangle=U_{1}\left|\frac{N}{2}\right\rangle$ used in Fig. 2. For TACT and TNT, $r$ was chosen to maximize $F_{Q}$ for $N=100$, while for OAT, a moderate value of $r$ was chosen such that the state was no longer in the spin-squeezed regime [54] but not sufficient to reach the maximum QFI spin-cat state, which occurs at $r=\frac{\pi}{2}$.

\begin{tabular}{lcc}
\hline \hline Scheme: & \multicolumn{1}{c}{$U_{1}$} & $r$ \\
\hline OAT & $e^{i r \hat{f}_{z}^{2}} e^{i \frac{\pi}{2} \hat{\jmath}_{y}}$ & 0.2 \\
TACT & $e^{i r\left(\hat{J}_{x}^{2}-\hat{\delta}_{y}^{2}\right)}$ & 0.032 \\
TNT & $e^{i r\left(\hat{S}_{z}^{2}-\frac{N}{2} \hat{J}_{x}\right)} e^{i \frac{\pi}{2} \hat{J}_{y}}$ & 0.0715 \\
Cat & $e^{i r \hat{\delta}_{z}^{2}} e^{i \frac{\pi}{2} \hat{y}_{y}}$ & $\frac{\pi}{2}$ \\
QPT & $\mathcal{T} \exp \left(\frac{-i}{\hbar} \int_{0}^{t_{0}} \hat{H}\left(t^{\prime}\right) d t^{\prime}\right) e^{i \frac{\pi}{2} \hat{J}_{y}}$ & \\
\hline \hline
\end{tabular}

application of $U_{\text {opt }}$ does not effect the CFI in the absence of noise; the Hellinger distance

$$
d_{H}^{2}=1-\sum_{m} \sqrt{P_{m}(\phi) P_{m}(\phi+\delta \phi)}
$$

is identical in Figs. 3(a) and 3(b) $\left(d_{H} \approx 0.24\right)$. However, $U_{\text {opt }}$ does effect how distinguishable the states remain after the addition of detection noise: $d_{H} \approx 0.067$ and 0.201 for Figs. 3(e) and 3(f) respectively.

\section{APPROACHING THE NQCRB WITH OAT-BASED IBRS}

While our optimum IBR gives us insight into what maximizes robustness, it is of no use to us unless we can find a physical mechanism with which it can be implemented. However, we can construct an IBR which has similar properties to the ideal case with the OAT mechanism. The OAT unitary can

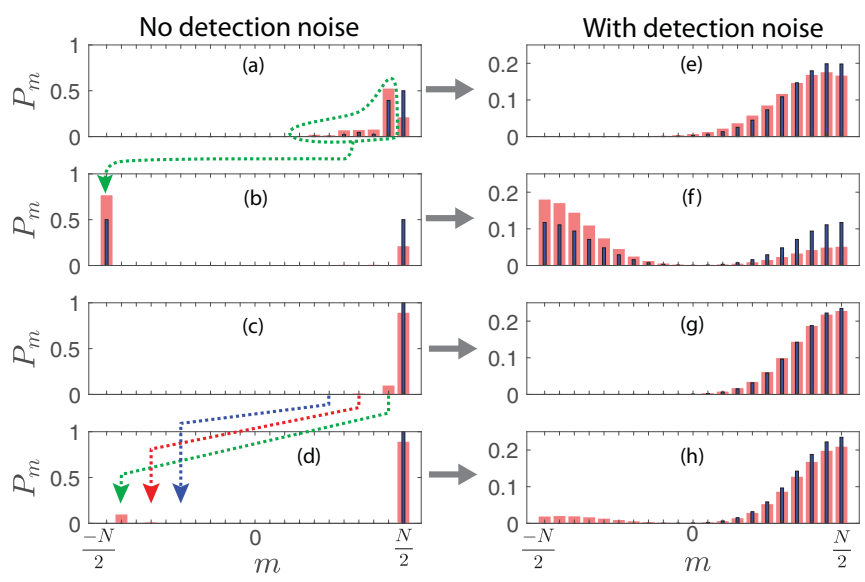

FIG. 3. $P_{m}(\phi)$ (blue thin bars) and $P_{m}(\phi+\delta \phi)$ (pink thick bars) with (right column) and without (left column) detection noise $\sigma$. [(a), (e)] $U_{2}=U_{1}^{\dagger}, \phi=\phi_{0}$. [(b), (f)] $U_{2}=U_{\text {opt }}, \phi=\phi_{0}$. [(c), (g) $]$ $U_{2}=U_{1}^{\dagger}, \phi=0$. [(d), (h)] $U_{2}=U_{\text {flip }} U_{1}^{\dagger}, \phi=0$. The Hellinger distance $d_{H}$ is [(a)-(d)] 0.238, (e) 0.067, (f) 0.201, (g) 0.012, and (h) 0.232. Parameters: $N=20, \sigma=3, r=0.2, \delta_{\phi}=\frac{1}{N}, \phi_{0}=0.118$. The behavior of $U_{\mathrm{p}}$ and $U_{\text {flip }}$ is indicated by the arrows between panels (a) and (b) and panels (c) and (d), respectively. 
be used to create the well-known spin-cat state $[73,74]$ :

$$
e^{i \frac{\pi}{2} \hat{J}_{y}^{2}}|m\rangle=\frac{1}{\sqrt{2}} e^{i \frac{\pi}{4}}\left(|m\rangle+i(-1)^{m}|-m\rangle\right) \equiv|\beta(m)\rangle,
$$

for even $N .^{1}$ This state has the unusual property that $\left|\left\langle\beta(m)\left|e^{i \frac{\pi}{2} \hat{J}_{z}}\right| \beta(m)\right\rangle\right|^{2}=\cos ^{2} \frac{m \pi}{2}$. That is, even- $m$ states are unaffected by a $\pi$ rotation, while odd- $m$ states become orthogonal. As such, a $\frac{\pi}{2}$ phase shift followed by secondary application of $e^{i \frac{\pi}{2} \hat{\delta}_{y}^{2}}$ will return $|\beta(m)\rangle$ to $|m\rangle$ if $m$ is even, or transfer it to an orthogonal state if $m$ is odd. Specifically,

$$
e^{i \frac{\pi}{2} \hat{J}_{y}^{2}} e^{i \frac{\pi}{2} \hat{J}_{z}} e^{i \frac{\pi}{2} \hat{J}_{y}^{2}}=-\sum_{m} i^{m(m-1)}\left|-1^{m} m\right\rangle\langle m| \equiv U_{\text {flip }} .
$$

The action of $U_{\text {flip }}$ is to exchange the odd elements of $P_{m}$ with $P_{-m}$, while leaving the even elements unaffected, as illustrated in Figs. 3(d) and 3(h). ${ }^{2}$ For sufficiently small $\phi$, most of the CFI for the state $U_{1}^{\dagger}\left|\psi_{\phi}\right\rangle$ is usually contained in the elements $m=\frac{N}{2}$ and $m=\frac{N}{2}-1$ [Figs. 3(c) and 3(g)]. Applying $U_{\text {flip }}$ to this state transfers probability from $m=\frac{N}{2}-1$ to $m=$ $-\left(\frac{N}{2}-1\right)$, forming a distribution almost as robust as $P_{\mathrm{opt}}$.

Figure 2 shows the performance of this scheme compared to $U_{\text {opt }}$ for quantum enhanced states generated via OAT, TACT, and TNT (see Table I). In these three cases, we see that $U_{2}=U_{\text {flip }} U_{1}^{\dagger}$ is very close to the optimum case $\left(U_{2}=U_{\mathrm{opt}}\right.$ and the NQCRB) and achieves sensitivity very close to the QCRB for detection noise $\sigma$ significantly exceeding $\sqrt{N}$. For comparison, we have also included the previously considered case of an echo, where $U_{2}=U_{1}^{\dagger}$, which performs significantly better than the case of no IBR $\left(U_{2}=U_{\theta}\right.$, where only a linear rotation is used to maximize the CFI), but not nearly as well as $U_{2}=U_{\text {flip }} U_{1}^{\dagger}$. We have also included the special case of OAT with $r=\frac{\pi}{2}$, which corresponds to the maximum QFI spin-cat state. In this case, both $U_{2}=U_{\text {flip }} U_{1}^{\dagger}$ and $U_{2}=U_{1}^{\dagger}$ saturate the NQCRB, while the case of no IBR loses all quantum enhancement for $\sigma \lesssim 1$. The reason why there is no need for the extra application of $U_{\text {flip }}$ is because the state $U_{1}^{\dagger}\left|\psi_{\phi}\right\rangle$ already yields a probability distribution identical to $P_{\text {opt }}$ and is unchanged by application of $U_{\text {flip }}$. The outstanding performance of the echo IBR for this state was first reported in Ref. [60] and subsequently in Refs. [61,65], but it was not known that this is the maximum achievable sensitivity. ${ }^{3}$

We also considered QPT, where the increased QFI is generated by slowly varying the parameters in a time-dependent Hamiltonian, such that the ground state is adiabatically transformed to one with high QFI. We implemented this with a Hamiltonian of the form

$$
\hat{H}=\hbar \chi\left(\hat{J}_{x} \cos ^{2} \frac{\pi}{2} \frac{t}{t_{0}}+\hat{J}_{z}^{2} \sin ^{2} \frac{\pi}{2} \frac{t}{t_{0}}\right)
$$

\footnotetext{
${ }^{1}$ For odd $N$, we require an additional rotation: an equal superposition cat is generated by $e^{i \frac{\pi}{2} \hat{J}_{y}} e^{i \frac{\pi}{2} \hat{J}_{y}^{2}}|m\rangle$.

${ }^{2}$ For odd $N$, an IBR that performs the same function is given by $U_{\text {flip }}=e^{i \frac{\pi}{2} \hat{J}_{y}\left(\hat{J}_{y}+1\right)} e^{i \theta \hat{J}_{z}} e^{i \frac{\pi}{2} \hat{J}_{y}\left(\hat{J}_{y}+1\right)}$, with $\theta=\frac{\pi}{2}(1+1 / N)$.

${ }^{3}$ We note that Ref. [61] reports higher robustness than this. However, the state is identical, and the discrepancy is due to a different convention for the detection noise.
}

such that

$$
U_{1}=\mathcal{T}\left[\exp \left(\frac{-i}{\hbar} \int_{0}^{t_{0}} \hat{H}\left(t^{\prime}\right) d t^{\prime}\right)\right] e^{i \frac{\pi}{2} \hat{J}_{y}},
$$

where $\mathcal{T}$ represents the time-ordering operator. In the limit $\chi t_{0} \rightarrow \infty, U_{1}\left|\frac{N}{2}\right\rangle=|0\rangle$, the twin-Fock state. We chose a moderate value $\chi t_{0}=20$, such that the final state contains nonzero elements on either side of $m=0$. Unlike the previous examples, when making measurements on the state $U_{1}^{\dagger}\left|\psi_{\phi}\right\rangle$ for small $\phi$, most of the CFI is contained in the elements $m=\frac{N}{2}$ and $m=\frac{N}{2}-2$, such that $U_{\text {flip }}$ has little effect. This is easily rectified, however, by using a modified IBR with $U_{\text {flip }}^{\prime}=$ $e^{i \frac{\pi}{2} \hat{\jmath}_{y}^{2}} e^{i \frac{\pi}{4} \hat{J}_{z}} e^{i \frac{\pi}{2} \hat{\jmath}_{y}^{2}}$, which for $N \gg 1, U_{\text {flip }}|m\rangle \approx|-m\rangle$ if $m / 2$ is odd. We see in Fig. 2(e) that this IBR is very close to the NQCRB.

The benefit of our IBR is not limited to pure states. We consider a quantum enhanced mixed state

$$
\rho=\sum_{m} e^{-\frac{m^{2}}{\Delta^{2}}}|m\rangle\langle m| /\left(\sum_{m} e^{-\frac{m^{2}}{\Delta^{2}}}\right) .
$$

We chose $\Delta=1$, which corresponds to a state with significant quantum enhancement, yet is far from pure, with the purity $\gamma=\operatorname{Tr}\left[\rho^{2}\right] \approx 0.4$. Such a state may arise from quantum enhancement via a strong QND interaction with a detuned optical field, as described in Ref. [75], with an imperfect measurement leading to uncertainty in $m$. Unlike the previous states considered, this state is mixed, so there is no unitary operator that maps this distribution to $P_{\mathrm{opt}}$. However, at $\phi=$ 0 , the final distribution is similar to the QPT case, which inspires us to use the same IBR, namely $U_{2}=U_{\text {flip }} U_{1}^{\dagger}$, with $U_{1}$ generated via the adiabatic evolution considered in the QPT example. We see in Fig. 2(f) that while this case is not as robust as previous examples, the general trend is the same; that is, $U_{2}=U_{\text {flip }} U_{1}^{\dagger}$ is more robust than $U_{2}=U_{1}^{\dagger}$, which in turn outperforms $U_{2}=U_{\theta}$. As the state is mixed, we cannot systematically construct $U_{\text {opt }}$. For completeness, we have also investigated applying our IBR to states with no quantum enhancement, such as coherent spin states [76], and find qualitatively similar results. ${ }^{4}$

\section{DISCUSSION}

The results of this paper may form an integral part of future quantum-enhanced sensing technologies, as high-QFI states are particularly susceptible to detection noise. While OAT-based quantum enhancement schemes are not yet capable of manufacturing spin-cat states (and therefore $U_{\text {flip }}$ ), progress in this area is rapid, particularly in schemes based on optically induced nonlinearities $[6,18]$ and Rydberg atoms [77]. Furthermore, we have provided insight and a systematic approach for constructing a robust IBR. Armed with this insight, schemes that approximate our optimum scheme may be found through other dynamical mechanisms that are perhaps easier to implement in a particular system. For example, it has

\footnotetext{
${ }^{4}$ The plot of $F_{C}(\sigma)$ for the coherent spin state is provided in the Supplementary Material [72].
} 
been shown that QPT can be used to engineer spin-cat states [46], so could potentially be used to construct a near-optimum IBR. One might question the wisdom of using an IBR that requires the ability to create a maximum QFI cat state in cases where the QFI of the input state is less than this. However, there may be situations when it is impractical to use a state preparation capable of creating a cat state, such as when the preparation time is limited [63]. Similarly, a state with less quantum enhancement may be desirable in the presence of external phase noise. In these situations, the presence of unavoidably large detection noise will still necessitate the use of a high-performance IBR in order to achieve high sensitivity. Finally, the NQCRB provides a limit for the performance of all IBR's. Once the sensitivity approaches this limit, further gains can only be made through the reduction of detection noise, rather than via improvement of the IBR.

\section{ACKNOWLEDGMENTS}

We acknowledge fruitful discussions with Samuel Nolan, Safoura Mirkhalaf, Luca Pezze, Augusto Smerzi, Manuel Gessner, and Jacob Dunningham. This work was supported by the European Union's Horizon 2020 research and innovation programme under the Marie Sklodowska-Curie Grant Agreement No. 704672.
[1] A. D. Cronin, J. Schmiedmayer, and D. E. Pritchard, Optics and interferometry with atoms and molecules, Rev. Mod. Phys. 81, 1051 (2009).

[2] L. Pezzè, A. Smerzi, M. K. Oberthaler, R. Schmied, and P. Treutlein, Quantum metrology with nonclassical states of atomic ensembles, arXiv:1609.01609.

[3] J. Esteve, C. Gross, A. Weller, S. Giovanazzi, and M. K. Oberthaler, Squeezing and entanglement in a Bose-Einstein condensate, Nature (London) 455, 1216 (2008).

[4] J. Appel, P. J. Windpassinger, D. Oblak, U. B. Hoff, N. Kjaergaard, and E. S. Polzik, Mesoscopic atomic entanglement for precision measurements beyond the standard quantum limit, Proc. Natl. Acad. Sci. USA 106, 10960 (2009).

[5] I. D. Leroux, M. H. Schleier-Smith, and V. Vuletić, Implementation of Cavity Squeezing of a Collective Atomic Spin, Phys. Rev. Lett. 104, 073602 (2010).

[6] M. H. Schleier-Smith, I. D. Leroux, and V. Vuletić, Squeezing the collective spin of a dilute atomic ensemble by cavity feedback, Phys. Rev. A 81, 021804 (2010).

[7] M. H. Schleier-Smith, I. D. Leroux, and V. Vuletić, States of an Ensemble of Two-Level Atoms with Reduced Quantum Uncertainty, Phys. Rev. Lett. 104, 073604 (2010).

[8] C. Gross, T. Zibold, E. Nicklas, J. Estève, and M. K. Oberthaler, Nonlinear atom interferometer surpasses classical precision limit, Nature (London) 464, 1165 (2010).

[9] M. F. Riedel, P. Böhi, Y. Li, T. W. Hänsch, A. Sinatra, and P. Treutlein, Atom-chip-based generation of entanglement for quantum metrology, Nature (London) 464, 1170 (2010).

[10] B. Lücke, M. Scherer, J. Kruse, L. Pezze, F. Deuretzbacher, P. Hyllus, O. Topic, J. Peise, W. Ertmer, J. Arlt et al., Twin matter waves for interferometry beyond the classical limit, Science 334, 773 (2011).

[11] C. D. Hamley, C. S. Gerving, T. M. Hoang, E. M. Bookjans, and M. S. Chapman, Spin-nematic squeezed vacuum in a quantum gas, Nat. Phys. 8, 305 (2012).

[12] T. Berrada, S. van Frank, R. Bücker, T. Schumm, J. F. Schaff, and J Schmiedmayer, Integrated Mach-Zehnder interferometer for Bose-Einstein condensates, Nat. Commun. 4, 2077 (2013).

[13] C. F. Ockeloen, R. Schmied, M. F. Riedel, and P. Treutlein, Quantum Metrology with a Scanning Probe Atom Interferometer, Phys. Rev. Lett. 111, 143001 (2013).

[14] H. Strobel, W. Muessel, D. Linnemann, T. Zibold, D. B. Hume, L. Pezzè, A. Smerzi, and M. K. Oberthaler, Fisher information and entanglement of non-Gaussian spin states, Science 345, 424 (2014).

[15] W. Muessel, H. Strobel, D. Linnemann, D. B. Hume, and M. K. Oberthaler, Scalable Spin Squeezing for QuantumEnhanced Magnetometry with Bose-Einstein Condensates, Phys. Rev. Lett. 113, 103004 (2014).

[16] W. Muessel, H. Strobel, D. Linnemann, T. Zibold, B. Juliá-Díaz, and M. K. Oberthaler, Twist-and-turn spin squeezing in BoseEinstein condensates, arXiv:1507.02930.

[17] I. Kruse, K. Lange, J. Peise, B. Lücke, L. Pezzè, J. Arlt, W. Ertmer, C. Lisdat, L. Santos, A. Smerzi, and C. Klempt, Improvement of an Atomic Clock Using Squeezed Vacuum, Phys. Rev. Lett. 117, 143004 (2016).

[18] O. Hosten, N. J. Engelsen, R. Krishnakumar, and M. A. Kasevich, Measurement noise 100 times lower than the quantum-projection limit using entangled atoms, Nature (London) 529, 505 (2016).

[19] Y.-Q. Zou, L.-N. Wu, Q. Liu, X.-Y. Luo, S.-F. Guo, J.-H. Cao, M. K. Tey, and L. You, Beating the classical precision limit with spin-1 Dicke state of more than 10000 atoms, PNAS 115, 6381 (2018).

[20] P. Hyllus, O. Gühne, and A. Smerzi, Not all pure entangled states are useful for sub-shot-noise interferometry, Phys. Rev. A 82, 012337 (2010)

[21] P. Hyllus, W. Laskowski, R. Krischek, C. Schwemmer, W. Wieczorek, H. Weinfurter, L. Pezzé, and A. Smerzi, Fisher information and multiparticle entanglement, Phys. Rev. A 85, 022321 (2012).

[22] G. Tóth, Multipartite entanglement and high-precision metrology, Phys. Rev. A 85, 022322 (2012).

[23] G. S. Agarwal and R. R. Puri, Cooperative behavior of atoms irradiated by broadband squeezed light, Phys. Rev. A 41, 3782 (1990).

[24] A. Kuzmich, K. Mølmer, and E. S. Polzik, Spin Squeezing in an Ensemble of Atoms Illuminated with Squeezed Light, Phys. Rev. Lett. 79, 4782 (1997).

[25] M. G. Moore, O. Zobay, and P. Meystre, Quantum optics of a Bose-Einstein condensate coupled to a quantized light field, Phys. Rev. A 60, 1491 (1999).

[26] H. Jing, J.-L. Chen, and M.-L. Ge, Quantum-dynamical theory for squeezing the output of a Bose-Einstein condensate, Phys. Rev. A 63, 015601 (2000).

[27] M. Fleischhauer and S. Gong, Stationary Source of Nonclassical or Entangled Atoms, Phys. Rev. Lett. 88, 070404 (2002). 
[28] S. A. Haine and J. J. Hope, Outcoupling from a Bose-Einstein condensate with squeezed light to produce entangled-atom laser beams, Phys. Rev. A 72, 033601 (2005).

[29] S. A. Haine and J. J. Hope, A multi-mode model of a nonclassical atom laser produced by outcoupling from a BoseEinstein condensate with squeezed light, Laser Phys. Lett. 2, 597 (2005).

[30] S. A. Haine, M. K. Olsen, and J. J. Hope, Generating Controllable Atom-Light Entanglement with a Raman Atom Laser System, Phys. Rev. Lett. 96, 133601 (2006).

[31] S. S. Szigeti, B. Tonekaboni, W. Yung S. Lau, S. N. Hood, and S. A. Haine, Squeezed-light-enhanced atom interferometry below the standard quantum limit, Phys. Rev. A 90, 063630 (2014).

[32] S. A. Haine, S. S. Szigeti, M. D. Lang, and C. M. Caves, Heisenberg-limited metrology with information recycling, Phys. Rev. A 91, 041802 (2015).

[33] A. Kuzmich, N. P. Bigelow, and L. Mandel, Atomic quantum non-demolition measurements and squeezing, Europhys. Lett. 42, 481 (1998).

[34] A. Kuzmich, L. Mandel, and N. P. Bigelow, Generation of Spin Squeezing Via Continuous Quantum Nondemolition Measurement, Phys. Rev. Lett. 85, 1594 (2000).

[35] A. Louchet-Chauvet, J. Appel, J. J Renema, D. Oblak, N. Kjaergaard, and E. S Polzik, Entanglement-assisted atomic clock beyond the projection noise limit, New J. Phys. 12, 065032 (2010).

[36] K. Hammerer, A. S. Sørensen, and E. S. Polzik, Quantum interface between light and atomic ensembles, Rev. Mod. Phys. 82, 1041 (2010).

[37] L.-M. Duan, A. Sørensen, J. I. Cirac, and P. Zoller, Squeezing and Entanglement of Atomic Beams, Phys. Rev. Lett. 85, 3991 (2000).

[38] H. Pu and P. Meystre, Creating Macroscopic Atomic EinsteinPodolsky-Rosen States from Bose-Einstein Condensates, Phys. Rev. Lett. 85, 3987 (2000).

[39] S. P. Nolan, J. Sabbatini, M. W. J. Bromley, M. J. Davis, and S. A. Haine, Quantum enhanced measurement of rotations with a spin-1 Bose-Einstein condensate in a ring trap, Phys. Rev. A 93, 023616 (2016).

[40] M. Kitagawa and M. Ueda, Squeezed spin states, Phys. Rev. A 47, 5138 (1993).

[41] A. S. Sørensen and K. Mølmer, Entanglement and Extreme Spin Squeezing, Phys. Rev. Lett. 86, 4431 (2001).

[42] S. A. Haine, J. Lau, R. P. Anderson, and M. T. Johnsson, Selfinduced spatial dynamics to enhance spin squeezing via oneaxis twisting in a two-component Bose-Einstein condensate, Phys. Rev. A 90, 023613 (2014).

[43] J. Ma and X. Wang, Fisher information and spin squeezing in the Lipkin-Meshkov-Glick model, Phys. Rev. A 80, 012318 (2009).

[44] C. K. Law, H. T. Ng, and P. T. Leung, Coherent control of spin squeezing, Phys. Rev. A 63, 055601 (2001).

[45] C. Lee, Adiabatic Mach-Zehnder Interferometry on a Quantized Bose-Josephson Junction, Phys. Rev. Lett. 97, 150402 (2006).

[46] C. Lee, Universality and Anomalous Mean-Field Breakdown of Symmetry-Breaking Transitions in a Coupled Two-Component Bose-Einstein Condensate, Phys. Rev. Lett. 102, 070401 (2009).
[47] Z. Zhang and L.-M. Duan, Generation of Massive Entanglement Through an Adiabatic Quantum Phase Transition in a Spinor Condensate, Phys. Rev. Lett. 111, 180401 (2013).

[48] H. Xing, A. Wang, Q.-S. Tan, W. Zhang, and S. Yi, Heisenbergscaled magnetometer with dipolar spin-1 condensates, Phys. Rev. A 93, 043615 (2016).

[49] X.-Y. Luo, Y.-Q. Zou, L.-N. Wu, Q. Liu, M.-F. Han, M. K. Tey, and L. You, Deterministic entanglement generation from driving through quantum phase transitions, Science 355, 620 (2017).

[50] P. Feldmann, M. Gessner, M. Gabbrielli, C. Klempt, L. Santos, L. Pezzè, and A. Smerzi, Interferometric sensitivity and entanglement by scanning through quantum phase transitions in spinor Bose-Einstein condensates, Phys. Rev. A 97, 032339 (2018).

[51] J. Huang, M. Zhuang, and C. Lee, Non-Gaussian precision metrology via driving through quantum phase transitions, Phys. Rev. A 97, 032116 (2018).

[52] R. Demkowicz-Dobrzanski, J. Kolodynski, and M. Guta, The elusive Heisenberg limit in quantum-enhanced metrology, Nat. Commun. 3, 1063 (2012).

[53] R. Demkowicz-Dobrzański, M. Jarzyna, and J. Kołodyński, Quantum limits in optical interferometry, Prog. Opt. 60, 345 (2015).

[54] E. Davis, G. Bentsen, and M. Schleier-Smith, Approaching the Heisenberg Limit Without Single-Particle Detection, Phys. Rev. Lett. 116, 053601 (2016).

[55] O. Hosten, R. Krishnakumar, N. J. Engelsen, and M. A. Kasevich, Quantum phase magnification, Science 352, 1552 (2016).

[56] F. Fröwis, P. Sekatski, and W. Dür, Detecting Large Quantum Fisher Information with Finite Measurement Precision, Phys. Rev. Lett. 116, 090801 (2016).

[57] T. Macrì, A. Smerzi, and L. Pezzè, Loschmidt echo for quantum metrology, Phys. Rev. A 94, 010102 (2016).

[58] D. Linnemann, H. Strobel, W. Muessel, J. Schulz, R. J. LewisSwan, K. V. Kheruntsyan, and M. K. Oberthaler, QuantumEnhanced Sensing Based on Time Reversal of Nonlinear Dynamics, Phys. Rev. Lett. 117, 013001 (2016).

[59] S. S. Szigeti, R. J. Lewis-Swan, and S. A. Haine, PumpedUp SU(1,1) Interferometry, Phys. Rev. Lett. 118, 150401 (2017).

[60] S. P. Nolan, S. S. Szigeti, and S. A. Haine, Optimal and Robust Quantum Metrology Using Interaction-Based Readouts, Phys. Rev. Lett. 119, 193601 (2017).

[61] R. Fang, R. Sarkar, and S. Shahriar, Enhancing sensitivity of an atom interferometer to the Heisenberg limit using increased quantum noise, arXiv: 1707.08260.

[62] F. Anders, L. Pezzè, A. Smerzi, and C. Klempt, Phase magnification by two-axis countertwisting for detection-noise robust interferometry, Phys. Rev. A 97, 043813 (2018).

[63] A. J Hayes, S. Dooley, W. J Munro, K. Nemoto, and J. Dunningham, Making the most of time in quantum metrology: Concurrent state preparation and sensing, Quantum Sci. Technol. 3, 035007 (2018).

[64] S. S. Mirkhalaf, S. P. Nolan, and S. A. Haine, Robustifying twist-and-turn entanglement with interaction-based readout, Phys. Rev. A 97, 053618 (2018). 
[65] J. Huang, M. Zhuang, B. Lu, Y. Ke, and C. Lee, Achieving Heisenberg-limited metrology with spin cat states via interaction-based readout, Phys. Rev. A 98, 012129 (2018).

[66] R. J. Lewis-Swan, M. A. Norcia, J. R. K. Cline, J. K. Thompson, and A. M. Rey, Robust Spin Squeezing Via Photon-Mediated Interactions on an Optical Clock Transition, Phys. Rev. Lett. 121, 070403 (2018).

[67] M. J. Holland and K. Burnett, Interferometric Detection of Optical Phase Shifts at the Heisenberg Limit, Phys. Rev. Lett. 71, 1355 (1993).

[68] V. Giovannetti, S. Lloyd, and L. Maccone, Quantum Metrology, Phys. Rev. Lett. 96, 010401 (2006).

[69] M. Gabbrielli, L. Pezzè, and A. Smerzi, Spin-Mixing Interferometry with Bose-Einstein condensates, Phys. Rev. Lett. 115, 163002 (2015).

[70] B. Yurke, S. L. McCall, and J. R. Klauder, SU(2) and SU(1,1) interferometers, Phys. Rev. A 33, 4033 (1986).

[71] L. Pezzé and A. Smerzi, Ultra-Sensitive Two-Mode Interferometry with Single-Mode Number Squeezing, Phys. Rev. Lett. 110, 163604 (2013).
[72] See Supplemental Material at http://link.aps.org/supplemental/ 10.1103/PhysRevA.98.030303 for further details of the derivation of Eq. (7), the optimization method, and these quantum states. By "quantum-enhanced states," we mean "states with $F_{Q}>N$."

[73] G. S. Agarwal, R. R. Puri, and R. P. Singh, Atomic Schrödinger cat states, Phys. Rev. A 56, 2249 (1997).

[74] S. P. Nolan and S. A. Haine, Quantum Fisher information as a predictor of decoherence in the preparation of spincat states for quantum metrology, Phys. Rev. A 95, 043642 (2017).

[75] S. A. Haine and S. S. Szigeti, Quantum metrology with mixed states: When recovering lost information is better than never losing it, Phys. Rev. A 92, 032317 (2015).

[76] J. M. Radcliffe, Some properties of coherent spin states, J. Phys. A 4, 313 (1971).

[77] H. Busche, P. Huillery, S. W. Ball, T. Ilieva, M. P. A. Jones, and C. S. Adams, Contactless nonlinear optics mediated by long-range Rydberg interactions, Nat. Phys. 13, 655 (2017). 